\title{
The physiological and disturbing effects of surgical face masks in the COVID-19 era
}

\author{
Dirol $\mathrm{H}^{1}$, Alkan $\mathrm{E}^{2}$, Sindel $\mathrm{M}^{2}$, Ozdemir $\mathrm{T}^{1}$, Erbas $\mathrm{D}^{3}$ \\ Chest Disease Department, Faculty of Medicine, Akdeniz University, Akdeniz University, Campus, \\ Antalya, Turkey. hulyadirol@akdeniz.edu.tr
}

\begin{abstract}
BACKGROUND: In the COVID-19 pandemic, the concern about mask-harmful effects disturbed maskadherence. However, it is not certain whether the masks cause cardiopulmonary overload.

OBJECTIVE: To investigate the physiological and disturbing effects of surgical face masks during exercise. METHOD: The study was conducted in a tertiary hospital with 100 healthy volunteers between September 2020 and January 2021. Individuals with impaired walking, cardiopulmonary disease, and smoking were not included in the study. Initially, respiratory rate (RR), heart rate (HR), oxygen saturation $\left(\mathrm{SpO}_{2}\right)$, end-tidal carbon dioxide $\left(\mathrm{EtCO}_{2}\right)$ were measured. Participants underwent 6-minute walking test (6MWT) with and without surgical masks. Mask-discomfort questionnaire was applied before and after 6 MWT with the mask. RESULTS: Surgical masks during 6 MWTs significantly increased HR, RR, and EtCO levels $(p<0.001)$. Walking distance $(p<0.001)$ and $\mathrm{SpO}_{2}$ level $(p=0.002)$ were significantly decreased with mask. In MaskDiscomfort Questionnaire, humidity, temperature, resistance, salinity, odor, fatigue $(p<0.001)$, and itching $(p=0.001)$ scores significantly increased after 6MWT with mask.

CONCLUSION: In healthy volunteers, $\mathrm{HR}, \mathrm{RR}, \mathrm{EtCO}_{2}$ were increased, and $\mathrm{SpO}_{2}$ and walking distance were decreased in the short-term, light exercise performed with the surgical mask. Findings support the concern that masks may cause cardiopulmonary overload (Tab. 3, Fig. 1, Ref. 17). Text in PDF www.elis.sk KEY WORDS: COVID-19, masks, physiology, psychological side effects, questionnaire.
\end{abstract}

\section{Introduction}

COVID-19 is a virus from coronaviridae family with a singlestranded RNA, an envelope, and protein protrusions on its surface (1). The transmission from person-to-person is mostly through droplets that come out of the mouth during coughing, sneezing, or talking (2). The virus was first detected in Wuhan in China and spread all over the world from there. Still, all countries of the world are fighting seriously with COVID-19 and applying strict restrictions to reduce the transmission rate. One of these important restrictions is the mandatory face mask use in the community. However, masks make our lives difficult, especially during exercise. In addition, many people do not want to use them because of fears about that masks may be harmful to their health and resist to this obligation. Based on concerns in the community about the harmful effects of masks, herein this study, we investigated the physiological and discomfort effects of surgical face masks during an exercise.

${ }^{1}$ Chest Disease Department, Faculty of Medicine, Akdeniz University Campus, Antalya, Turkey, ${ }^{2}$ Anatomy Department, Faculty of Medicine, Akdeniz University, Turkey, and ${ }^{3}$ Physiology Department, Faculty of Medicine, Kyrenia University, Northern Cyprus

Address for correspondence: H. Dirol, Chest Disease Department, Faculty of Medicine, Akdeniz University, Akdeniz University Dumlupinar Boulevard, Campus, Antalya, Postal zip code: 07058, Turkey.

Phone: +90.242.2493612, Fax: +90.242.2496040

\section{Method}

The study was conducted in the Department of Chest Diseases and the Department of Anatomy. Approval for the study was granted by the Clinical Research Ethics Committee of Akdeniz University Faculty of Medicine on 26.08.2020 (decision no: KAEK-652).

\section{The study group (Subjects)}

Volunteers $(\mathrm{n}=100)$, consisting of healthy individuals, participated in the study. Individuals with a medical history of impaired walking, cardiovascular or respiratory comorbidity and smokers were not included. Written informed consent was obtained from all participants by providing information about the study.

\section{Measurements}

The walking test for 6-minute (6MWT) was carried out in a 30-meter-long closed corridor with a hard floor according to the guidelines of the American Thoracic Society (ATS) (3). A distance was marked every 3 meters along the course, starting and ending points were also marked. In the study, $6 \mathrm{MWT}$ was performed twice for each individual, with and without a face mask and distances walked by participants were noted. Three-layer surgical masks were used in the study. Before 6MWT, the volunteers rested on a chair close to the corridor for 15 minutes. Body temperature from the forehead, blood pressure from the arm, heart rate, and 
oxygen saturation $\left(\mathrm{SpO}_{2}\right)$ with a pulse oximeter, respiratory rate, and $\mathrm{EtCO}_{2}$ with capnograph were measured before every 6MWT and immediately after every 6MWT. Participants were randomly assigned to the tests with the first 6MWT without a mask, the second 6MWT with a mask or vice versa, and they were allowed to rest for at least 30 minutes between two 6MWT tests. The test was initiated by instructing the volunteers to walk at a fast pace and safely along the course of the corridor, they were informed about the remaining time during the test but did not receive any motivation. Jumper Infrared Thermometer (JPD-FR202, Guangdong, CHINA) for body temperature, Erka Perfect Aneroid (München, GERMANY) for blood pressure, ChoiceMMed finger pulse oximeter (MD300C15D, Beijing, CHINA) for heart rate and $\mathrm{SpO}_{2}$ and Nonin Respsense capnograph (Amsterdam, Netherlands) for respiratory rate and $\mathrm{EtCO}_{2}$ were used. Each of the devices was calibrated before the measurements.

\section{Mask discomfort survey}

Since the mask discomfort questionnaire is aimed at the discomfort of the mask, this questionnaire was only applied before and after 6MWT with the mask. The questionnaire containing 10 questions, was used for the evaluation. In the survey, the moisture and the heat created by the mask on the face and the discomfort caused by the dryness and salinity in the mouth; discomfort caused by resistance due to the mask encountered during breathing; discomfort due to the incompatibility and tightness of the mask; discomfort caused by itching and odor due to the mask; the feeling of fatigue created by the mask and, on average, the uncomfortable feeling created by the mask on the person, were evaluated. Scoring
Tab. 1. Demographic data.

\begin{tabular}{lccccc}
\hline \multirow{2}{*}{ Sex } & \multicolumn{2}{c}{ Female } & \multicolumn{2}{c}{58} & \multirow{2}{*}{ Total n=100 } \\
\cline { 2 - 4 } & \multicolumn{2}{c}{ Male } & 42 & & \\
\hline & $\mathrm{n}$ & Mean & Std. Deviation & Min & Max \\
\hline Age (years) & & 40.87 & 12.73 & 20 & 65 \\
18-34 & 40 & 27.62 & 3.94 & & \\
35-65 & 60 & 49.70 & 7.96 & & 189 \\
\hline Height $(\mathrm{cm})$ & & 168.49 & 8.60 & 150 & 129 \\
\hline Weight $(\mathrm{kg})$ & & 72.49 & 13.82 & 46 & 36.50 \\
\hline BMI & & 26.55 & 3.24 & 17.10 & \\
$<20$ & 11 & 19.07 & 0.91 & & \\
$20-30$ & 78 & 25.27 & 2.60 & & \\
$>30$ & 11 & 33.24 & 1.83 & & \\
\hline
\end{tabular}

58 females and 42 males. total 100 participants' ages in years and numbers of participants aged below or over 35 -year subgroups, heights, weights and body mass index and number of participants belong in three subgroups, below 20, between 20 and 30, over 30 , given as mean values, standard deviation, minimum and maximum range.

ranged from 0 to $10 ; 0$ was considered as completely comfortable and 10 as completely uncomfortable.

\section{Statistical analysis}

All analyses were performed using the SPSS statistical software program (SPSS v.23, IBM, Somers, NY) and graphs made with GraphPad Prism 9 (v.9.0.0-121, San Diego, California, USA). With "repeated one-way ANOVA measurements ", it was tested whether the difference within subjects was significant. In order to use this analysis, normality assumption and homogeneous variance assumption should be provided. The assumption of normality has been provided in the study. Mauchly's W was used if the assump-
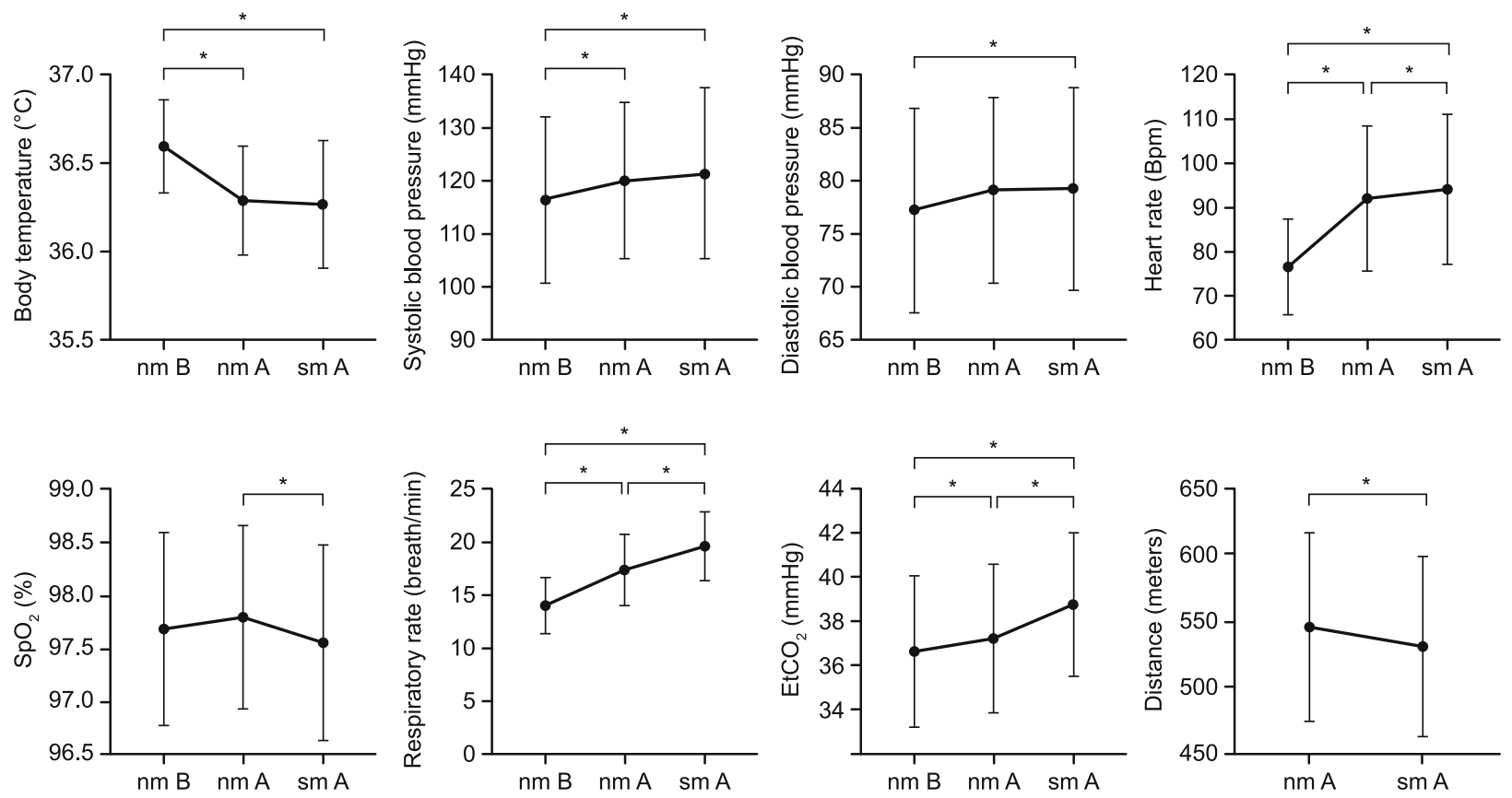

Fig. 1. Graphs of measurements in mean and standard deviation. nm B, no mask while resting before 6MWT; nm A, after 6MWT without mask; sm A, after 6MWT with surgical mask. Statistically significant results of paired samples t-test results are indicated with asterisk. 
Tab. 2. Measurement results of healthy volunteers' physiological parameters before and after 6 MWT.

\begin{tabular}{|c|c|c|c|c|c|c|c|}
\hline Parameter & $\begin{array}{c}\text { Before 6MWT } \\
(\mathrm{nm} \mathrm{B})\end{array}$ & $\begin{array}{l}\text { After 6MWT } \\
\text { (nm A) }\end{array}$ & $\begin{array}{c}\text { After 6MWT with } \\
\text { surgical mask (sm A) }\end{array}$ & ANOVA & $\begin{array}{c}\mathrm{nm} \mathrm{B} \mathrm{vs.} \\
\mathrm{nm} \mathrm{A}\end{array}$ & $\begin{array}{c}\mathrm{nm} \mathrm{B} \mathrm{vs.} \\
\mathrm{sm} \mathrm{A}\end{array}$ & $\begin{array}{c}\mathrm{nm} \text { A vs. } \\
\text { sm A }\end{array}$ \\
\hline Skin Temperature $\left({ }^{\circ} \mathrm{C}\right)$ & $36.59 \pm 0.26$ & $36.29 \pm 0.31$ & $36.27 \pm 0.36$ & $<0.001^{\mathrm{a}}$ & $<0.001$ & $<0.001$ & 0.493 \\
\hline Systolic Blood Pressure (mmHg) & $116.31 \pm 15.67$ & $119.99 \pm 14.72$ & $121.29 \pm 16.12$ & $<0.001^{\mathrm{a}}$ & $<0.001$ & $<0.001$ & 0.105 \\
\hline Diastolic Blood Pressure (mmHg) & $77.25 \pm 9.64$ & $78.50 \pm 10.92$ & $79.34 \pm 9.58$ & $0.069^{\mathrm{a}}$ & 0.195 & 0.001 & 0.397 \\
\hline Hearth Rate (beat/min) & $76.58 \pm 10.86$ & $91.47 \pm 18.27$ & $94.06 \pm 17.03$ & $<0.001^{\mathrm{b}}$ & $<0.001$ & $<0.001$ & 0.015 \\
\hline $\mathrm{SpO}_{2}(\%)$ & $97.69 \pm 0.91$ & $97.80 \pm 0.86$ & $97.56 \pm 0.92$ & $0.006^{\mathrm{a}}$ & 0.131 & 0.085 & 0.002 \\
\hline Respiratory Rate (resp/min) & $14.10 \pm 2.65$ & $17.46 \pm 3.31$ & $19.70 \pm 3.21$ & $<0.001^{\text {b }}$ & $<0.001$ & $<0.001$ & $<0.001$ \\
\hline Distance (meters) & & $545.20 \pm 71.23$ & $530.70 \pm 67.85$ & $<0.001^{\text {b }}$ & & & $<0.001$ \\
\hline
\end{tabular}

Wearing no mask before 6-minute walking test 6MWT (nm B), wearing no mask after 6MWT (nm A), wearing a surgical mask and 6MWT (sm A) given as mean \pm standard deviation. Within subjects repeated measures ANOVA results and paired samples t-test results of: no mask vs. no mask $6 \mathrm{MWT}$ (nm B vs. nm A). no mask vs. surgical mask 6MWT (nm B vs. sm A). no mask 6MWT vs. surgical mask 6MWT (nm A vs. sm A) given as p-values. Statically significant results (p $\leq 0.05$ ) are indicated in bold ${ }^{a}$ Mauchly's W Test of Sphericity assumed. ${ }^{\text {b}}$ Mauchly's W Test of Sphericity not assumed, used Greenhouse-Geisser correction $(\varepsilon<0.75)$.

tion of homogeneous variance was made, and Greenhouse-Geisser correction was used if it was not $(\varepsilon<0.75)$. Dependent variables with a significant $\mathrm{p}$-value were analyzed with the paired t-test with the Bonferroni correction again. In all analyses, a value of $\mathrm{p} \leq 0.05$ was considered statistically significant.

\section{Results}

The average age of 100 volunteers, 58 of whom were women, was 40.87 ( \pm 12.73$)$. Mean height was $168.49 \mathrm{~cm}( \pm 8.60)$, average weight $72.49 \mathrm{~kg}( \pm 13.82)$, body mass index was 26.55 ( \pm 3.24) (Tab. 1). Volunteers measurements were performed before and after each 6MWT, with and without the mask (Fig. 1).

Skin temperature decreased after 6MWT with and without the mask, and there was a significant difference compared to initial measurements without the mask (ANOVA $p<0.001$, paired t-test $\mathrm{p}<0.001$ ). Body temperature decreased by $0.303{ }^{\circ} \mathrm{C}$ and 0.322 ${ }^{\circ} \mathrm{C}$ after 6MWT without and with the mask, respectively. It was not significant ( $p \geq 0.05$ ) (Tab. 2).

For systolic blood pressure, there was a significant difference in "repeated ANOVA measurements " within the subject, while no significant difference was found for diastolic blood pressure $(\mathrm{p}<$ 0.001 and $p \geq 0.05$, respectively). Systolic blood pressure increased by $3.68 \mathrm{mmHg}$ and $4.98 \mathrm{mmHg}$ after 6MWT, with and without the

Tab. 3. Mask-Discomfort survey results of healthy volunteers.

\begin{tabular}{lcccc}
\hline Parameter & Before & After & $\mathrm{p}$ & $\begin{array}{c}\text { Difference } \\
\text { Percentage }\end{array}$ \\
\hline Moisture & $4.74 \pm 2.64$ & $6.33 \pm 2.63$ & $\mathbf{0 . 0 0 0}$ & $33.54 \%$ \\
Heat & $4.44 \pm 2.54$ & $6.28 \pm 2.62$ & $\mathbf{0 . 0 0 0}$ & $41.44 \%$ \\
Resistance & $4.38 \pm 2.48$ & $5.68 \pm 2.71$ & $\mathbf{0 . 0 0 0}$ & $29.68 \%$ \\
Itchiness & $1.97 \pm 2.87$ & $2.45 \pm 2.99$ & $\mathbf{0 . 0 0 1}$ & $24.37 \%$ \\
Tightness & $3.75 \pm 3.12$ & $3.92 \pm 3.06$ & 0.065 & $4.53 \%$ \\
Saltiness & $1.90 \pm 2.44$ & $3.25 \pm 3.17$ & $\mathbf{0 . 0 0 0}$ & $71.05 \%$ \\
Incompatibility & $3.49 \pm 3.18$ & $3.55 \pm 3.22$ & 0.441 & $1.72 \%$ \\
Odor & $3.70 \pm 3.30$ & $4.55 \pm 3.62$ & $\mathbf{0 . 0 0 0}$ & $22.97 \%$ \\
Fatigue & $3.15 \pm 2.87$ & $4.64 \pm 3.21$ & $\mathbf{0 . 0 0 0}$ & $47.30 \%$ \\
Overall discomfort & $5.18 \pm 1.99$ & $6.70 \pm 2.17$ & $\mathbf{0 . 0 0 0}$ & $29.47 \%$ \\
\hline
\end{tabular}

Before and after 6-minute walking test while wearing surgical mask given as mean \pm standard deviation. Paired samples t-test results of each parameter given as p-values. Statistically significant results $(\mathrm{p} \leq 0.05)$ are indicated in bold mask compared to initial measurements but no significant difference was found between groups after 6MWT ( $p \geq 0.105$ ) (Tab. 2).

Heart rate increased by an average of 14.89 and 17.48 after $6 \mathrm{MWT}$ without the mask and with the mask respectively, and the effect of the mask on heart rate was found statistically significant (ANOVA $\mathrm{p}<0.001)$.

The respiratory rate increased to 3.36 and 5.60 after 6MWT, without and with the mask, respectively and the mask's effect was statistically significant (ANOVA $<<0.001)$. Respiratory rate after $6 \mathrm{MWT}$ without and with the mask was found to be significantly important at $\mathrm{p}<0.001$.

The distance was $545.20 \pm 71.23$ and $530.70 \pm 67.85$ meters after $6 \mathrm{MWT}$ without and with the mask, respectively. The mean distance after 6MWT decreased by 14.5 meters because of the mask, and it was significant $(\mathrm{p}<0.001)$.

$\mathrm{SpO}_{2}$ increased by an average of $0.11 \%$ after $6 \mathrm{MWT}$ without the mask but decreased by an average of $0.13 \%$ after $6 \mathrm{MWT}$ with the mask and it was statistically significant $(\mathrm{p} \leq 0.05)$. There was no significant difference between the mean values of initial measurements compared to the after 6MWT with the mask and without the mask ( $p \geq 0.05$ and $p \geq 0.05$, respectively). But it was significant between the groups after $6 \mathrm{MWT}$ with and without the mask $(\mathrm{p} \leq 0.05)$.

$\mathrm{EtCO}_{2}$ values increased by $0.61 \mathrm{mmHg}$ and by $2.13 \mathrm{mmHg}$ after 6MWT without and with the mask, respectively. The efficacy of the mask was statistically significant (ANOVA $p<0.001$ ). The paired t-test also showed a significant difference between groups after 6MWT with and without the mask $(\mathrm{p}<0.001)$.

\section{Mask Discomfort Survey}

The results of the t-test analysis depending on the mean values and standard deviations of the scores given by the volunteers of the discomfort questionnaire are given in Table 3.

Paired t-test analysis between the pre and post 6MWT questionnaire with mask scores showed a significant difference $(\mathrm{p}<$ 0.001 ) for humidity, temperature, resistance, salinity, odor, fatigue, and itching.

No significant difference was found between pre and post 6MWT questionnaires for discomfort scores caused by the tightness and incompatibility of the mask ( $p \geq 0.05)$. 
Overall discomfort score was significant between pre and post 6MWT questionnaire with mask $(\mathrm{p}<0.001)$.

\section{Discussion}

In this study, we found that surgical face mask use during exercise caused a perception of serious discomfort, and this subjective situation was accompanied by some physiological changes. The overall discomfort score calculated after the exercise with the mask increased significantly compared to pre-exercise. Exercising with a surgical face mask increased the heart rate, respiratory rate, and $\mathrm{EtCO}_{2}$ values more than exercise without the mask. In addition, $\mathrm{SpO}_{2}$ values and walking distance decreased significantly during exercise with mask compared to the exercise with no mask. Masks during exercise had no significant effect on skin temperature and blood pressure.

Masks are protective against droplet-based disease and are generally well tolerated. In the COVID-19 pandemic, their importance to the control of the epidemic cannot be ignored. However, concerns about the potential harmful effect of mask use led to the development of resistance and negative effect for its use. It is known that the surgical mask, which is recommended to be used in daily outdoor activities during the pandemic, causes discomfort due to the heat and moisture retention effect. There are also increasing clues that different masks cause significant physiological changes in different workloads, even in healthy people. Although the mask is an effective method to prevent transmission, we should be aware of the physiological changes it may cause. We believe that it is the most rational way to improve the existing masks, to minimize the negative physiological effects, to increase comfort and adherence.

Masks cause respiratory resistance. It is difficult to breathe against this resistance. Comfort is one of the most important factors providing mask adherence. In addition to breathing difficulties, masks also cause a feeling of incompatibility, fatigue, and general discomfort on the face (4). Breathing difficulty may also vary depending on the type and workload performed during the mask use. Surgical masks also cause discomfort, but it is less than N95 masks (5). In our study, the use of masks during exercise significantly increased the overall discomfort score. Participants felt significantly more heat, moisture, salinity, fatigue, respiratory resistance, itching, and odor during the exercise with the mask. Besides these complaints, the participants found the masks to be equally tight and incompatible with their faces before and after exercise. The discomfort and its severity that we have evaluated with the questionnaire are based on the subjective statements of the participants. In addition to the subjective perception of discomfort, masks affect the heart and respiratory system, and those may also contribute to the feeling of discomfort.

One of the physiological variables we examined was skin temperature. Skin temperature is kept constant by the contribution of many physiological mechanisms. The face is important in the regulation of body temperature. Skin thermal receptors are particularly concentrated on the face $(6-8)$. When the face of a healthy individual is heated, three times more local sweating oc- curs over the legs than the leg sweating that occurs when the leg is heated $(6,9)$ By creating a microclimate inside the mask, it can affect the whole-body thermal perception (10). The use of a surgical mask during the operation increases the facial temperature by $5{ }^{\circ} \mathrm{C}(11)$. In another study, it was found that perioral skin temperature increased after using a surgical mask (12). Despite these findings, respirators used for pesticide spraying by agricultural workers did not cause temperature changes in the edge of the mouth and nose (13). Such different results regarding the effect of the mask on body temperature may be due to the type of mask and the weight and length of the work done with the mask. In our study, body temperature decreased after exercise with and without the surgical masks compared to pre-exercise. Skin temperature may have been decreased by hyperventilation or sweating in response to the increase in heat nearby the mask. We concluded that while our body temperature decreased with the use of masks during exercise, our heat perception increased.

Heart rate increased with the use of surgical masks during steady-state exercise (14). In another study, heart rate was found to be unchanged with the use of a respirator (13). In our study, heart rate increased significantly at the end of the exercise in both groups compared to the levels before the exercise and the heart rateincrease in the group with the mask was significantly higher than in the group without the mask. Due to the increased oxygen need during exercise, an increase in heart rate is an expected condition. This increase was greater with mask and maybe a compensatory response of the cardiovascular system to the increase in airway resistance and respiratory workload. While healthy people can compensate for the increase in respiratory workload by increasing the heart rate during exercise with the mask, this compensation may not occur in patients with cardiac comorbidity or increased cardiac workload may cause additional problems.

Lässing et al (14) investigated the effect of the surgical face mask during steady-state exercise, and they found that the mask reduced the forced expiratory volume in one second, vital capacity, and peak expiratory flow while increasing the airway resistance. In another study, respiratory function tests showed a reduction in ventilation, cardiopulmonary exercise capacity, and comfort with both surgical masks and respirators (15). There is little data about the mask effect on the blood gases. The mask creates an additional dead space just around the mouth and nose as the continuation of the nasal and oral cavity. It is speculated carbondioxide may accumulate while oxygen may decrease in the inner side of the mask. The $\mathrm{SpO}_{2}$ levels of surgeons with surgical masks decreased significantly in prolonged surgical procedures. In another study, most of the patients using N95 during 4-hour hemodialysis had decreased $\mathrm{pO}_{2}$ and increased respiratory rate (16). In our study, using a surgical face mask during exercise resulted in an increase in the respiratory rate and a decrease in $\mathrm{SpO}_{2}$ compared to exercise without a mask. Compensatory responses may not be enough in heavier and longer-lasting activities or those with heart and lung comorbidities. A decrease of $\mathrm{SpO}_{2}$ or an increase of $\mathrm{EtCO}_{2}$ may be more pronounced. Vigorous exercises performed by wearing the N95 or face mask may be harmful and may pose some health risks, therefore, it was recommended to 
exercise at low or medium intensity instead of vigorous exercise while wearing a face mask (17).

Our study has some limitations. We could not perform the body plethysmograph and cardiopulmonary exercise tests during the first phase of the pandemic. Our study population is young people with no comorbidities, and results may change at older ages and in case of comorbidity. Although we conducted all tests in the same environment, temperature and humidity may have differed slightly. The diet and fluid deficits of the participants could not be evaluated on the exercise day. Standardization on clothing could not be achieved due to contamination concerns, but the participants were advised to wear sports shoes and comfortable clothes.

\section{Conclusion}

The study demonstrated that there is a significant increase in heart rate, respiratory rate, $\mathrm{EtCO}_{2}$, and a significant decrease in $\mathrm{SpO}_{2}$ and walking distance during exercise with a surgical face mask. The perception of humidity, temperature, resistance, salinity, odor, fatigue, and itching increased significantly after the exercise with the mask. Our findings support the concerns that exercise with a surgical mask may impose a cardiac and pulmonary extra-load, even in elders with cardiopulmonary disease these effects may turn to a cardiopulmonary overload and decompensation.

\section{References}

1. Pal M, Berhanu G, Desalegn C, Kandi V. Severe Acute Respiratory Syndrome Coronavirus-2 (SARS-CoV-2): An Update. Cureus 2020; 12 (3): e7423.

2. Dhand R, Li J. Coughs and Sneezes: Their Role in Transmission of Respiratory Viral Infections, Including SARS-CoV-2. Am J Respir Crit Care Med 2020; 202 (5): 651-659.

3. Laboratories ATSCoPSfCPF. ATS statement: guidelines for the sixminute walk test. Am J Respir Crit Care Med 2002; 166 (1): 111-117.

4. Barrett KE, Barman SM, Boitano S et al. Ganong's review of medical physiology2016.
5. Li Y, Tokura H, Guo YP et al. Effects of wearing N95 and surgical facemasks on heart rate, thermal stress and subjective sensations. Int Arch Occup Environ Health 2005; 78 (6): 501-509.

6. Nadel ER, Mitchell JW, Stolwijk JA. Differential thermal sensitivity in the human skin. Pflugers Arch 1973; 340 (1): 71-76.

7. Nakamura M, Yoda T, Crawshaw LI et al. Relative importance of different surface regions for thermal comfort in humans. Eur J Appl Physiol 2013; 113 (1): 63-76.

8. Stevens JC, Choo KK. Temperature sensitivity of the body surface over the life span. Somatosens Mot Res 1998; 15 (1): 13-28.

9. Cotter JD, Taylor NA. The distribution of cutaneous sudomotor and alliesthesial thermosensitivity in mildly heat-stressed humans: an openloop approach. J Physiol 2005; 565 (Pt 1): 335-345.

10. Lim EC, Seet RC, Lee KH, Wilder-Smith EP, Chuah BY, Ong BK. Headaches and the N95 face-mask amongst healthcare providers. Acta neurologica Scandinavica 2006; 113 (3): 199-202.

11. Enerson DM, Eisenfeld LI, Kajikuri H. Heat and moisture trapping beneath surgical face masks: a consideration of factors affecting the surgeon's discomfort and performance. Surgery 1967; 62 (6): 1007-1016.

12. Scarano A, Inchingolo F, Lorusso F. Facial Skin Temperature and Discomfort When Wearing Protective Face Masks: Thermal Infrared Imaging Evaluation and Hands Moving the Mask. Internat J Environment Res Publ Health 2020; 17 (13).

13. Laird IS, Goldsmith R, Pack RJ, Vitalis A. The effect on heart rate and facial skin temperature of wearing respiratory protection at work. Ann Occupat Hygiene 2002; 46 (2): 143-148.

14. Lässing J, Falz R, Pökel C et al. Effects of surgical face masks on cardiopulmonary parameters during steady state exercise. Sci Reports 2020; 10 (1): 22363.

15. Fikenzer S, Uhe T, Lavall D et al. Effects of surgical and FFP2/ N95 face masks on cardiopulmonary exercise capacity. Clinical research in cardiology: official journal of the German Cardiac Society 2020; 109 (12): $1522-1530$.

16. Kao TW, Huang KC, Huang YL, Tsai TJ, Hsieh BS, Wu MS. The physiological impact of wearing an N95 mask during hemodialysis as a precaution against SARS in patients with end-stage renal disease. J Formosan Med Ass 2004; 103 (8): 624-628.

17. Chandrasekaran B, Fernandes S. Exercise with facemask; Are we handling a devil's sword? A physiological hypothesis. Med Hypotheses 2020; 144: 110002 . 